\title{
Challenges to Managing Virtual Teams in Georgian SMEs
}

\author{
Anna Ābeltinga ${ }^{1 *}$ and Ketevan Rizhamadze ${ }^{2}$ \\ ${ }^{1}$ D.Sc. (Economics), Associate Professor, EKA University of Applied Science, Rīga, LV-1019, \\ Latvia \\ ${ }^{2}$ MBus (Global Business Analysis), Doctoral student, Turiba University, Riga LV-1058, Latvia
}

\begin{abstract}
The novel coronavirus pandemic has brought about an unprecedented economic and social crisis. The work methods have seen a significant change, and telework has experienced swift growth. The practical application of teleworking needs technology, social and organizational support. Employees who are less tech-savvy require digital training. There is limited data on this topic. Therefore, this research will contribute to a deeper understanding of the issue related to managing virtual teams. An objective of this study is to examine how virtual teams are developed and to identify the challenges to managing virtual reams in selected Georgian SMEs. This study seeks to obtain data that will help to analyze and review issues that arise in the process of managing diverse and geographically dispersed virtual teams. This research is constructed on a brief literature review, survey and focus group discussions among the representatives of SMEs operating in the financial and IT sector in Georgia. According to study findings, companies that pursue successful eleadership consider teleworking as an opportunity. The most interesting finding is that timely availability of necessary information, effective communication and well-defined tasks influence the collaboration between virtual team members and is pivotal whilst managing a virtual team.
\end{abstract}

\section{Introduction}

The leading characteristic of modern society tends to be the presence of organizations. The existence of organizations goes back to older civilizations. Today, many organizations are performing every task virtually to ensure the effective functioning of society [1]. Increasing innovation and speediness to market and being updated with local market conditions and requirements make it crucial to utilize virtual teams. Companies embark on a virtual team tactic to their work for the following reasons: mergers and acquisitions, looking for resources in other markets, the potential for growth worldwide and attracting talent from abroad. Although virtual teams can be faster, more innovative and flexible, they require coordinated management, organization and support. The internalization of economic and industrial settings, the globalized business environment has caused human re-localization.

* Corresponding author: anna.abeltina $@$ gmail.com

Corresponding author: katerizhamadze@gmail.com 
Fierce competition shortened the product development lifecycle. Consequently, international organizations are using virtual networks quite frequently for cost efficiency [2]. The massive shock of the coronavirus pandemic has hugely affected every aspect of ordinary life. According to data published by World Bank, the international economy is in a recession [3]. The new reality reshaped the way we work, interact and communicate with customers. In times of uncertainty, creativity and innovation are essential for companies and showing empathy and authenticity to customers and suppliers tends to be pivotal. The new standard has forced managers to think more about the new ways of keeping their teams motivated, productive and supportive [4]. To deal with challenges many organizations have established virtual teams. The term "virtual team" implies that individuals from different departments gather to solve a specific aim and objective. Thus, team leaders are responsible for promptly establishing an operational team. In addition, managers must create the proper context and organizational structure for the group [5]. People are separated by distance or time in virtual groups. Hitherto have joint tasks to perform and shared aims to achieve. The team must attain the identical outcomes as traditional co-located teams, nonetheless from their detached sites. Virtual teams often facilitate collaboration. By bringing together individuals from various backgrounds, the organization is more willing to satisfy the fastchanging market demands. Audio and video conferencing tools play an essential role in the functioning of the virtual teams [6]. Teams are interdependent and coordinate toward a common goal. Even though the roles and responsibilities differ among team members, they still hold the same objective and work together towards achieving that goal. Members that are geographically diversified rely on digital technologies heavily, and communication is achieved through technologies. Coordination, communication and collaboration in virtual teams differ from the traditional teams. Units can coordinate work and perform well when they share the knowledge and experience [7]. However, for groups that do not work, virtual teams are not a panacea. In contrast, the management of virtual teams is more challenging than the traditional team.

The investigation is organized in the following way. It commences by laying out the theoretical dimensions of the research and looks at how virtual teams are managed from a global perspective. The next chapter is concerned with the methodology used for this study and research question, hypothesis, goals and methods are given. The last section presents the findings of the research and concludes the study.

\section{Theoretical background}

Virtual teams are successful when team members possess appropriate skills and behaviors. Independent people, collective goals and multiple media is needed to launch a virtual squad [8]. A project manager must have a good understanding of leadership theory and must make clear distinction between leadership and communication. In addition, there might be some differences between the management of virtual and traditional teams [9]. Duarte and Snyder proposed recommendations on how to manage virtual teams (see Table 1):

Table 1. Mastering virtual teams: Strategies, tools, and techniques

\begin{tabular}{|l|l|}
\hline $\begin{array}{l}\text { Properly functioning } \\
\text { information system }\end{array}$ & $\begin{array}{l}\text { The organization has fully functioning } \\
\text { information systems. The organization has } \\
\text { employees who have digital literacy skills. }\end{array}$ \\
\hline $\begin{array}{l}\text { Software updates on } \\
\text { personal computers }\end{array}$ & $\begin{array}{l}\text { The organization constantly updates and } \\
\text { monitors personal computer systems. }\end{array}$ \\
\hline Corporate network & $\begin{array}{l}\text { The corporate network is vital in the } \\
\text { organization. Consequently, more complex }\end{array}$ \\
\hline
\end{tabular}


systems within the organization can exist.

Source: Elaborated by authors based on Duarte and Snyder [14].

To overcome the cooperation problem, four attributes of a team can be highlighted [10]:

-Individuals are assigned to the team;

-Tasks are well defined;

-Common goals are established;

-Embeddedness is ensured.

Communication and information technologies play a crucial role in the functioning of virtual teams. Communication tends to be a vital element for any team. Clear communication is essential to all aspects of virtual teams [11]. The main aim of the communication process is to transmit information, where both sender and receiver share an understanding of the symbols. The characteristics of the receiver or audience is considered: using signs that are familiar to the receiver or audience. The selection of medium of transmission is essential. Virtual teams cannot succeed without innovation, and team-based innovation is necessary for its functioning [12]. Communication technologies that simplify the arduous work process and enable sharing of knowledge among team members must be heavily utilized [13]. As the results of Mehrabian's research reveal body language is a major factor in interpersonal communication, followed by the tone of voice and words (see Table 2).

Table 2: Mehrabian's communications model

\begin{tabular}{|l|l|}
\hline $\mathbf{5 5} \%$ & Body gesture \\
\hline $\mathbf{3 8} \%$ & Speech pattern \\
\hline $\mathbf{7 \%}$ & Expression \\
\hline
\end{tabular}

Source: Elaborated by authors based on Mehrabian's Rule [25].

Management of virtual teams is challenging and time-consuming. Trust building is complicated in a virtual environment and necessitates a thoughtful approach [14]. As Mehrabian's findings reveal, communication via body language is crucially important during interpersonal communications, which is sometimes missing and is tough to seize in a virtual team. Apart from communication, the most critical part of a relationship - trust is also challenging to build and maintain within online and virtual communication. Trust tends to be the foundation for a functional team [15]. The adaptation stage tends to be significant in every relationship. In addition, it becomes incredibly sturdy to undergo it in a virtual setting [16]. Previous studies have highlighted the significance of traditional interaction in building and establishing trust [17]. In addition, touch and human interaction are also important within the trust-building process, and it is achieved through direct interpersonal communications. It is complicated to showcase the friendliness, kindness and warmth in virtual teams [18]. In culturally diversified virtual teams, it is vital to create a global mindset [19]. Working in virtual teams requires dealing with national culture, unique culture and corporate culture. Understanding unique culture and then different cultures lead to finding the appropriate approach to build trust and commitment in virtual teams [20].

\section{Research question and research hypothesis}


The present research explores the management of virtual teams in Georgian SMEs. This study provides new insights into the main concepts of a virtual team and explores the problems. This research will identify the major obstacles that managers of SMEs face during the management of virtual teams. This prospective study was designed to scrutinize best practices and to issue advice and recommendation to managers and leaders of SMEs for ensuring the successful operation and functioning of virtual teams and remote work. Following research questions and hypotheses are formulated:

- Research Question 1: In the face of the epidemic's impact, remote work has become a norm, working from the office versus working from home: which is suitable for you?? Hypothesis 1: A combination of traditional and remote work

- Research Question 2: What are the most significant problems faced by SMEs during the management of teams?

Hypothesis 2: Building and establishing trust and clear communication

\section{Research methods}

This study used a combination of qualitative and quantitative research methods. A focus group was used for obtaining qualitative data, whereas an online survey was distributed for collecting quantitative information. Online survey was conducted by utilizing a convenience sample of 120 SMEs. Qualitative data were gathered via organizing an online focus group consisting of 12 participants in January 2021. The purpose of the research was to identify the challenges to managing virtual teams in Georgian SMEs in the financial and IT sector. The target population for the study was SMEs operating in Georgia in the field of finance (banking) and IT.

The discussion in the focus group was divided into three parts: the first part was related to the general industry information. The second part was concerned with the assessment of work style (remote vs office), and the third section addressed challenges while managing virtual teams. The focus group was monitored by a moderator who stimulated conversation among the contributors. A survey included multiple-choice, yes/no and ranking questions. The survey was short, and questions were clear and straightforward. The questionnaire was designed by using an online survey development website. Participation in the focus group and survey was entirely voluntary without giving monetary compensation to the participants.

\section{Research methods}

There was a time when some experts wondered if an effective work team was even possible if its members were not physically located together. In addition, it was argued that communication technologies could never become the substitute to face to face interpersonal interaction. Nowadays, we see that technology and the ubiquity of the internet bend people together who are dispersed geographically. Even though it is challenging to manage and lead a group of people virtually, it is still possible [21]. Following challenges can be identified within the management of virtual teams, as illustrated in Table 3:

Table 3. Significant challenges of virtual teams

\begin{tabular}{|c|}
\hline In-person communication \\
\hline Lack of resources \\
\hline Lack of training provided to staff \\
\hline The difference in time zone and location \\
\hline
\end{tabular}




\begin{tabular}{|c|}
\hline Involvement in more than one team \\
\hline Information is not well shared among team members \\
\hline
\end{tabular}

Source: Elaborated by authors based on DeRosa and Lepsinger (2010) [22].

As mentioned in the literature review, among the factors which influence on collaboration between virtual team members, adequate communication and timely availability of necessary information are highlighted by 50, 30 participants, respectively, as demonstrated in Table 4:

Table 4. Factors affecting the functioning of virtual teams

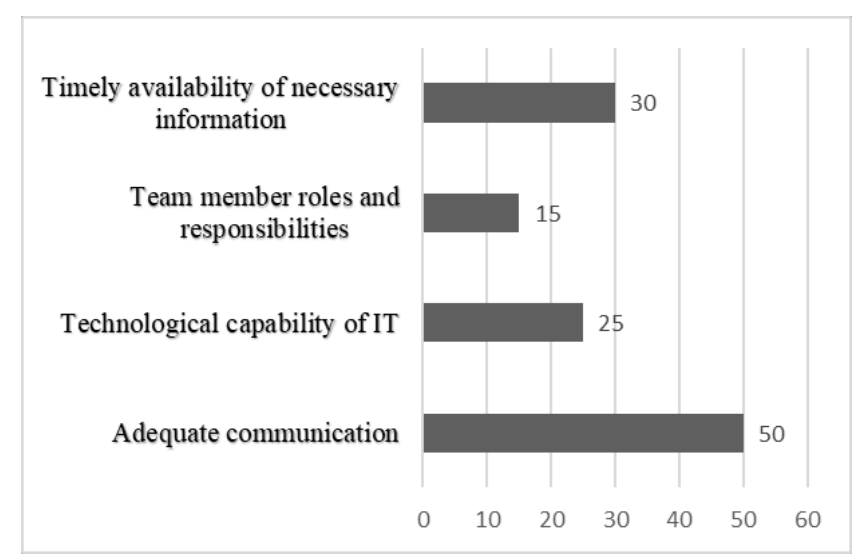

Source: Elaborated by authors

Prior studies have noted the importance of communication in trust-building process. In addition to previous studies, the results of this study did show that the majority of respondents believe that communication has an influence on trust- building between virtual team members, as shown in Table 5:

Table 5. The importance of communication in trust building in virtual teams

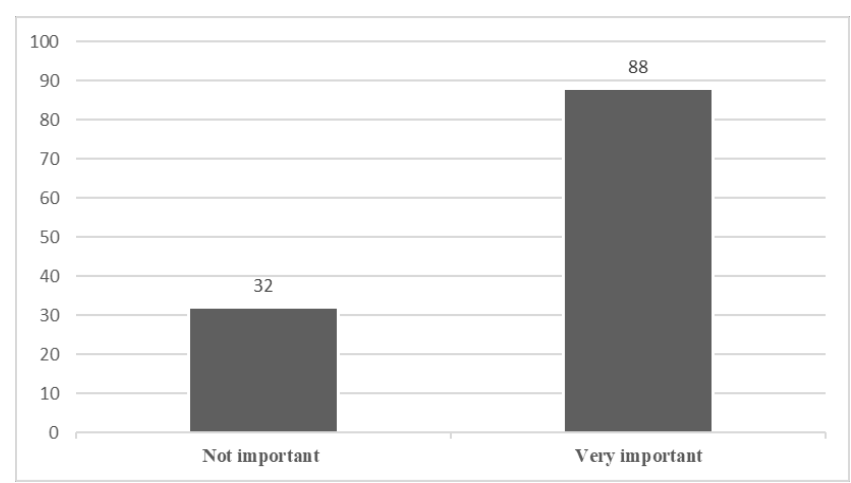

Source: Elaborated by authors

Concerning the first research question, it was hypothesized that a combination of traditional and remote work would be undertaken by the organizations. As the results illustrated in Table 6 indicate, these results seem to be consistent with the hypothesis: 
Table 6. Remote work vs office work

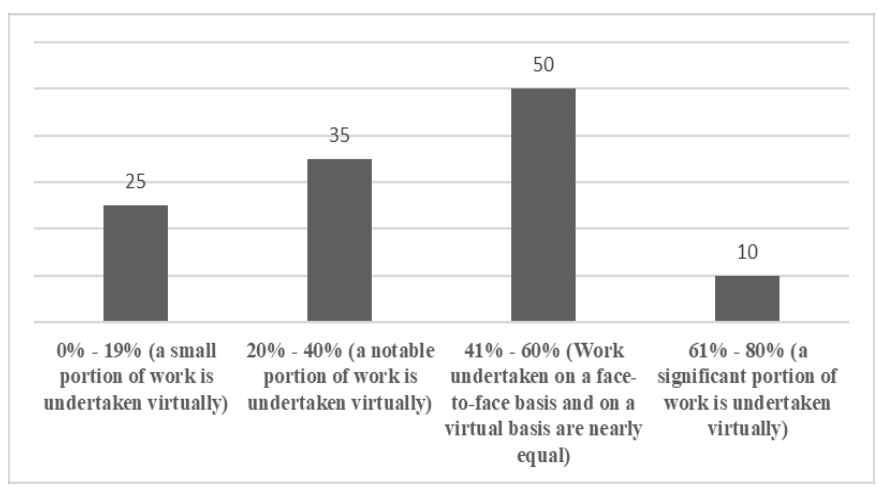

Source: Elaborated by authors

The results of this study suggest that video conference tools (52 replies) are heavily used in online communication. It is followed by emails ( 20 replies) and the project's website (10 replies) or extranet (10 $\mathrm{r}$ replies). The results are displayed in Table 7:

Table 7. Favorite video conferencing software

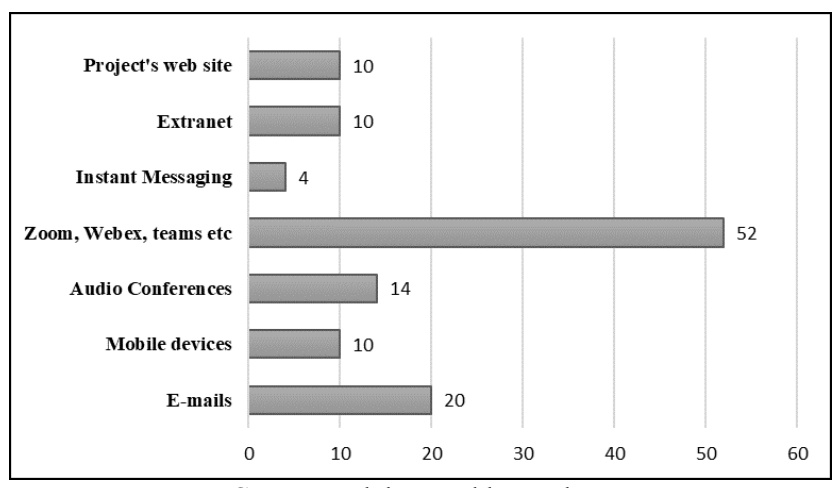

Source: Elaborated by authors

In reviewing the literature, we see that in virtual teams, building and maintaining trust among team members takes a longer time, and fewer opportunities are present to create social capital. Also, in the absence of visual cues and frequent communications, it is utterly arduous to understand cultural dissimilarities and languages. Therefore, a team leader has a considerable responsibility in ensuring credibility and competency without establishing authority among the team [23]. In addition, when team members actively participate in teambuilding activities and are involved in the organizational process the success is guaranteed. It is crucial to set up virtual teams so that each team member has to contribute something and everyone has equal opportunities to participate. In addition, it is important to 
provide time for a virtual team member to socialize casually after the activity or formal meeting [24].

The results are shown in Table 8 match those observed in earlier studies. Among the factors which have an influence on trust-building between virtual team members, the respondents of the survey have highlighted the following: task communication, defined communication patterns and transparent team leading:

Table 8. Factors influencing trust-building

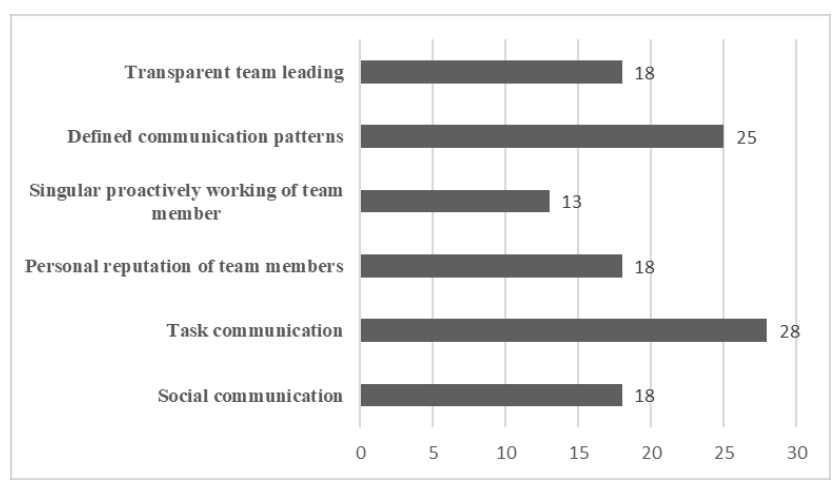

Source: Elaborated by authors

The most prominent finding to emerge from the analysis is that Covid-19 was the reason that forced the organizations to create virtual teams, as indicated in Table 9:

Table 9. The reasons for establishing virtual teams

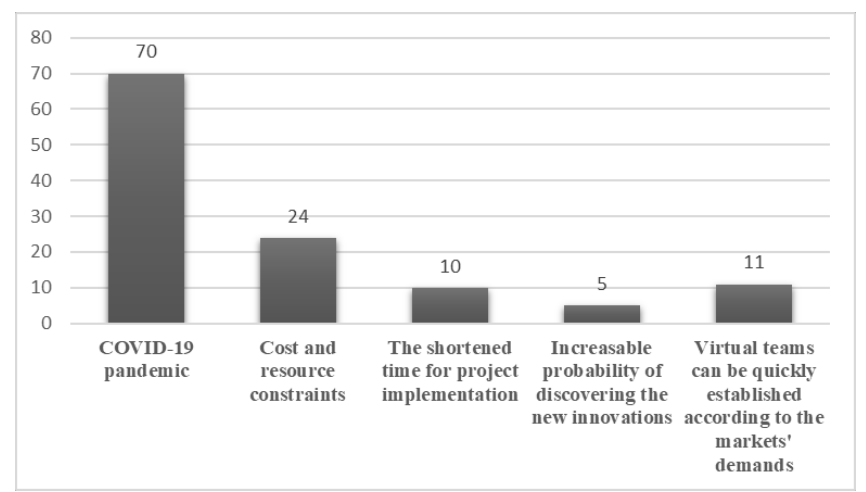

Source: Elaborated by authors

Regarding the focus group discussion, most participants prefer a combination and mixture of virtual and traditional work. Following are the communication tools frequently used to communicate between colleagues and video conferences: Emails, Zoom, Skype, Webex and Microsoft teams. Consistent with the literature, this research found that participants deem adequate communication and trust to be the most critical factors for proper functioning of a virtual team. This finding broadly supports the idea that employees require digital education and training. Some participants also emphasized the fact that the tasks among team members should be separated, and they should have a clear understanding of the roles and 
responsibilities. As per their discussion, the team member should have access to accurate information on a timely basis, and managers should possess the necessary skills to manage the team successfully. In addition, one of the challenges is that there is a lack of structure in virtual teams and having less connection and contact with colleagues can become a source of losing productivity, motivation and concentration.

\section{Conclusion}

In this rapidly changing environment and circumstances, we see the rise of virtual teams. Virtual teams tend to be the new normal. However, the switch from in-person interactions to online communication is a daunting task. An utterly distinctive method is required to manage virtual teams. As team members do not have a chance to have a side conversation, company culture is built virtually. Trust is pivotal in the work process as it creates a setting where people are willing to contribute. However, building trust in these settings is challenging. Subsequently, virtual team leaders have a role in guaranteeing that team members are equally presented and respected despite their backgrounds. Overall, this study strengthens the idea that it is essential to create a space where common goals and objectives are established. Taken together, these findings suggest that factors that affect the functioning of traditional teams are the same as when groups work remotely. Although the current study is based on a small sample of participants, the findings suggest that more time, energy and clear guidance from both leaders and workers are needed when dealing with virtual teams. Notwithstanding the relatively limited sample, this work offers valuable insights into the management of virtual teams in Georgian SMEs.

\section{References}

1. Afflerbach, T. (2020). Hybrid Virtual Teams in Shared Services Organizations: Practices to Overcome the Cooperation Problem. Cham, Switzerland: Springer.

2. Brennan, R., Canning, L. and McDowell, R. (2007) Business-to-Business marketing. London: SAGE publications ltd.

3. Brown, M. K., Huettner, B., \& James-Tanny, C. (2007). Managing virtual teams: Getting the most from wikis, blogs, and other collaborative tools: Managing virtual teams. Piano, TX: Wordware Pub.

4. Davis, D. D. (2004). The Tao of leadership in virtual teams. Organizational Dynamics, 33(1), 47-62.

5. DeRosa, D., \& Lepsinger, R., (2010). Virtual team success: A practical guide for working and leading from a distance. Jossey-Bass.

6. Duarte, D. L., \& Snyder, N. T. (2006). Mastering virtual teams: Strategies, tools, and techniques that succeed. San Francisco, CA: Jossey-Bass

7. Edwards, A., \& Wilson, J. R. (2004). Implementing virtual teams: A practical guide. Aldershot: Gower. P6

8. Fisher, K., \& Fisher, M. D. (2011). Manager's guide to virtual teams. New York: McGraw-Hill.

9. Gardenswartz, L. (2003). The global diversity desk reference: Managing an international workforce. San Francisco: Pfeiffer.

10. Geber, B. (1995). Virtual teams. Training. 32(4), 36-40. Journal of Management, 32(4). 
11. Gibson, C. B., \& Cohen, S. G. (2013). Virtual Teams That Work: Creating Conditions for Virtual Team Effectiveness. John Wiley \& Sons.

12. Ivanaj, S., \& Bozon, C. (2016.). Managing virtual teams. Edward Elgar Publication Limited.

13. Jarvenpaa, S. L., Knoll, K., \& Leidner, D. E. (1998). Is anybody out there? Antecedents of trust in global virtual teams. Journal of Management Information Systems, 14(4), 29-64. doi:10.1080/07421222.1998.11518185

14. Larson, C. E., \& J., L. F. (1990). Teamwork: What must go right, what can go wrong. Newbury Park u.a.: Sage.

15. Lee, M. R. (2014). Leading virtual project teams: Adapting leadership theories and communications techniques to $21^{\text {st }}$ century organizations. CRC press

16. Lencioni, P., \& Stransky, C. (2002). The Five Dysfunctions of a Team. NY: Random House.

17. Lipnack, J., \& Stamps, J. (1997). Virtual teams: Reaching across space, time, and organizations with technology. New York: John Wiley \&Sons

18. Lipnack, J., \& Stamps, J. (2000). Virtual teams: People working across boundaries with technology. New York: John Wiley \&Sons

19. Mehrabian, A., (1972). Nonverbal Communication. Aldine-Atherton, Chicago, Illinois

20. Pauleen, D. J. (2004). Virtual teams: Projects, protocols and processes. Hershey etc.: Idea Group Publishing

21. Price Waterhouse Coopers PWC, (2021). (Online). Retrieved on 15 February 2021 from https://www.pwc.com/sg/en/publications/a-resilient-tomorrow-covid-19response-and-transformation/keeping-your-virtual-team-together.html

22. Scott, W. R., \& Davis, G. F. (2017). Organizations and organizing: Rational, natural, and open systems perspectives. Oxford: Routledge. P2

23. Settle-Murphy, N. M. (2017). Leading effective virtual teams: Overcoming time and distance to achieve exceptional results. Boca Raton: Auerbach.

24. Siebdrat, F. Hoegl, M. Ernst, H. (2008). The bright side of virtual Collaboration: How teams can profit from dispersion. Academy of Management Proceedings, 2008(1), 1-6. doi:10.5465/ambpp.2008.33627369

25. World Bank (2020). COVID-19 to Plunge Global Economy into Worst Recession since World War II. (Online). Retrieved on 20 February 2021 from https://www.worldbank.org/en/news/press-release/2020/06/08/covid-19-to-plungeglobal-economy-into-worst-recession-since-world-war-ii 